\title{
Les structures argyrophiles tégumentaires des stades larvaires (miracidium, rédie, cercaire) de Fasciola bepatica. Comparaison avec F. gigantica.
}

\author{
par Ch. BAYSSADE-DUFOUR *, J.-L. ALBARET *, P. SAMNALIEV **, \\ J. CASSONE * et V. DIMITROV ** \\ * Muséum national d'Histoire naturelle, Laboratoire de Zoologie (Vers), \\ associé au C.N.R.S., 43, rue Cuvier, F 75231 Paris Cedex 05. \\ ** Bulgarian Academy of Sciences, Central Laboratory of Helminthology, \\ Sofia $1113 \mathrm{Kv}$ "Geo Milev» Akad. G. Bontchev str. bl. 3, Bulgaria.
}

RESUME. Les structures tégumentaires argyrophiles du miracidium et de la cercaire de Fasciola hepatica sont décrites et comparées à celles de F. gigantica.

Les miracidiums des deux espèces ont les organites du térébratorium et de la première ceinture identiques. Au contraire, la plaque ciliée médio-ventrale du deuxième étage diffère statistiquement selon l'espèce : elle est, ordinairement, plus longue que les autres plaques de cet étage chez $F$. hepatica et est plus souvent de la même taille chez F. gigantica.

Les cercaires des deux espèces ont un premier cycle céphalique identique et une chétotaxie corporelle peu différente. Par contre, les terminaisons sensorielles à deux niveaux céphaliques et sur la queue permettent de les différencier.

La chétotaxie d'une rédie-fille de $F$. hepatica est décrite; elle montre sur le pourtour buccal une forte concentration de sensilles, aux niveaux pharyngien et post-pharyngiens des sensilles plus espacées sur une face supposée ventrale et quelques rares sensilles sur l'autre face présumée dorsale.

Integumentary argentophilic structures in larval stages (miracidium, redia, cercaria) of Fasciola hepatica (Trematoda, Fasciolidae). Comparison with F. gigantica.

SUMMARY. Integumentary argentophilic structures of the miracidium and cercaria are described and with those of $\mathrm{F}$. gigantica compared.

Accepté le 15 avril 1980. 
In the miracidia, the pattern of the organites of the terebratorium and the arrangement of the papillae between the first and second tier of epidermal plates are similar for the two species. However, the disposition of the medio-ventral plate of the second tier allows the miracidia of the two related species to be distinguished statistically.

In the cercariae, the chaetotaxy differs between F. hepatica and F. gigantica in two cephalic regions and on the tail (presence of UD papillae in $\mathrm{F}$. hepatica, absence in F. gigantica).

The chaetotaxy of a daughter-redia of F. hepatica is also described. There is an important concentration of papillae around the buccal aperture, whereas at the pharyngeal and post pharyngeal levels, papillae are more spaced on the side supposed to be ventral, and scarce on the side considered dorsal.

Selon la bibliographie consacrée aux grandes douves du foie, Fasciola hepatica Linné, 1758, est classiquement considérée comme la douve des régions tempérées et F. gigantica Cobbold, 1855, comme celle des régions tropicales (Kendall, 1965). Toutefois, divers auteurs dont Bhalerao (1934), Popov (1957), Azimov et coll. (1972), Sahba et coll. (1972), Everaert et coll. (1974) signalent la présence simultanée des deux douves dans divers pays des cinq continents. Nasir et coll. (1968) signalent l'existence de cercaires des deux espèces au Vénézuela et donnent une clé de détermination basée sur la disposition de l'appareil digestif des cercaires. Boray (1966), Sahba et coll. (1972), Lin et coll. (1974), Sazanov (1977) énumèrent les espèces de mollusques permettant le développement de l'une ou l'autre douve et celles permettant le développement des deux. Porter (1938), comparant $F$. hepatica et $F$. gigantica, fait remarquer que la détermination peut s'avérer délicate et que les formes typiques de chaque espèce représentent les deux extrémités d'une longue série entre lesquelles beaucoup de formes intermédiaires se rencontrent.

Nous décrivons ici les stades larvaires : miracidiums et cercaires de $F$. hepatica, sachant que généralement l'imprégnation argentique de ces stades permet, chez les Trématodes, une diagnose très précise, et nous les comparons à ceux de $F$. gigantica. Nous décrivons aussi la chétotaxie d'une rédie-fille de $F$. hepatica.

\section{I. - Matériel et méthodes}

Deux collections de miracidiums proviennent respectivement d'œufs obtenus par dilacération de $F$. hepatica adultes, recueillies dans le foie d'un bœuf (Bos taurus) à l'abattoir de Versailles (France) et d'œufs obtenus, après incubation à $37^{\circ} \mathrm{C}$, de $F$. hepatica adultes recueillies dans le foie d'une vache à l'abattoir de Sofia ainsi que d'œufs obtenus par rinçage de la bile de cette même vache. 
La collection de Versailles comprend 66 miracidiums; celle de Sofia 84 miracidiums. Ces miracidiums sont imprégnés au nitrate d'argent selon la méthode de Lynch (1933). Les rédies sont obtenues par dissection de Lymnaea truncatula naturellement infestées, récoltées à $2 \mathrm{~km}$ du village de Novi Cham, à une vingtaine de kilomètres à l'est de Sofia (Bulgarie). Les cercaires sont émises par des Lymnaea truncatula naturellement infestées, récoltées près du village de Moussatchevo, à une vingtaine de kilomètres de Sofia. Rédies et cercaires sont imprégnées au nitrate d'argent à $2 \%$ selon la méthode de Combes, Bayssade-Dufour et Cassone (1976). Ce matériel est comparé aux miracidiums et cercaires de F. gigantica décrits par Albaret et coll. (1980) ; les miracidiums provenaient d'œufs obtenus par dilacération de $F$. gigantica adultes recueillies dans le foie de trois vaches N'Dama (Bos taurus) à l'abattoir de Kolda (Sénégal) ; les observations ont porté sur trois populations comprenant respectivement 35, 75 et 42 miracidiums; les cercaires correspondantes, donc de même origine que les miracidiums, avaient été obtenues expérimentalement par infestation de Lymnaea natalensis.

\section{II. - Description des stades larvaires de Fasciola bepatica.}

\section{Le miracidium.}

a. Plaques épidermiques ciliées (fig. 1 et fig. 6, A-C).

Les plaques ciliées, au nombre de 21 , sont réparties en cinq étages comprenant d'avant en arrière $6,6,3,4$ et 2 plaques séparées par quatre ceintures transversales. La plaque médio-ventrale du deuxième étage, observée chez deux populations de miracidiums, l'une originaire de Bulgarie ( 84 miracidiums), l'autre de France (66 miracidiums), montre une disposition susceptible de varier. Cette plaque se prolonge jusqu'à la ceinture 3 dans respectivement $62 \%$ et $82 \%$ des cas (fig. $1, A$ et B, et fig. 6 , $A$ ), elle s'interrompt au niveau de la ceinture 2 dans $18 \%$ et $9 \%$ des cas (fig. $1, C$ et fig. $6, C$ ) et elle occupe une position intermédiaire dans $20 \%$ et $9 \%$ des cas (fig. 1, D et fig. $6, B$ ). Cette variation permet statistiquement de différencier le miracidium de $F$. hepatica de celui de $F$. gigantica.

b. Organites argyrophiles superficiels (fig. 1, B, E, F).

Les organites argyrophiles superficiels sont portés sur le térébratorium et la première ceinture transversale. Leur nombre et leur disposition chez $F$. hepatica sont en tous points identiques à ceux du miracidium de $F$. gigantica décrits dans un travail récent (Albaret et coll., 1980).

\section{La rédie-fille (fig. 2).}

Les sensilles s'observent dans la région antérieure de la rédie-fille de $F$. hepatica. Il semble qu'elles puissent s'inscrire sur des cycles rappelant ceux des cercaires; elles sont très concentrées au niveau buccal où l'on peut admettre qu'elles accompagnent 

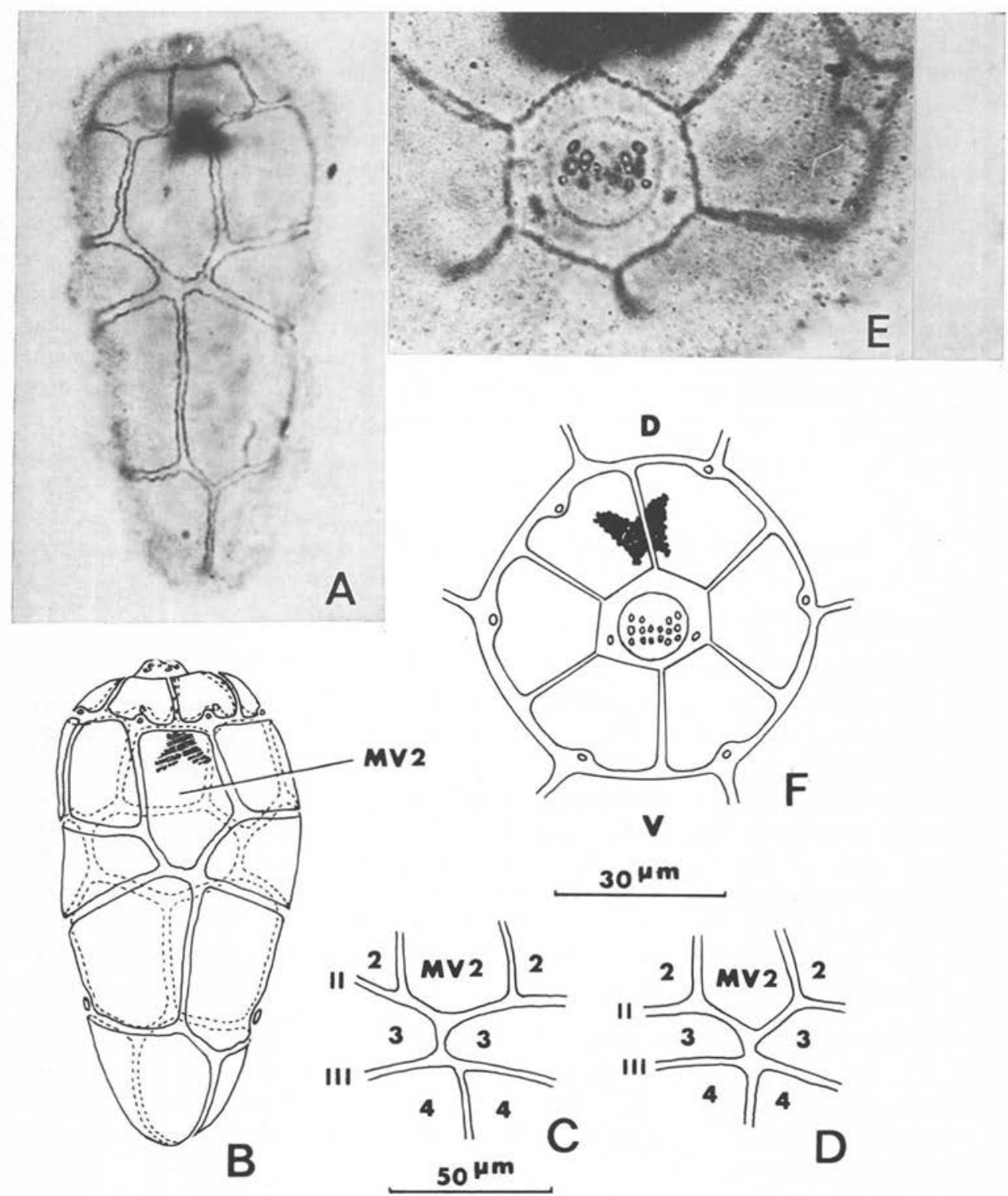

Fig. 1. Miracidium de $F$. hepatica. A et B : vues ventrales; A, imprégnation argentique $\times 470$; $\mathrm{C}$ et D, variations de la disposition de la plaque ciliée médio-ventrale (MV2) ; 2, 3, 4 plaques ciliées des deuxième, troisième et quatrième étages ; II et III : deuxième et troisième ceintures; E, vue apicale du térébratorium, imprégnation argentique $\times 1250 ; \mathrm{F}$, vue apicale du térébratorium et de la première ceinture; D : dorsal; V : ventral. 
un phénomène de céphalisation; dans la région pharyngienne et post-pharyngienne, elles se répartissent sur deux faces inégalement pourvues; par analogie avec le cas le plus fréquent chez les cercaires, nous supposons ventrale la face la plus riche en soies; la symétrie bilatérale qui devrait exister pour chaque face est imparfaite sur nos spécimens ; les sensilles nous ont paru absentes sur le reste du tégument de la rédie.
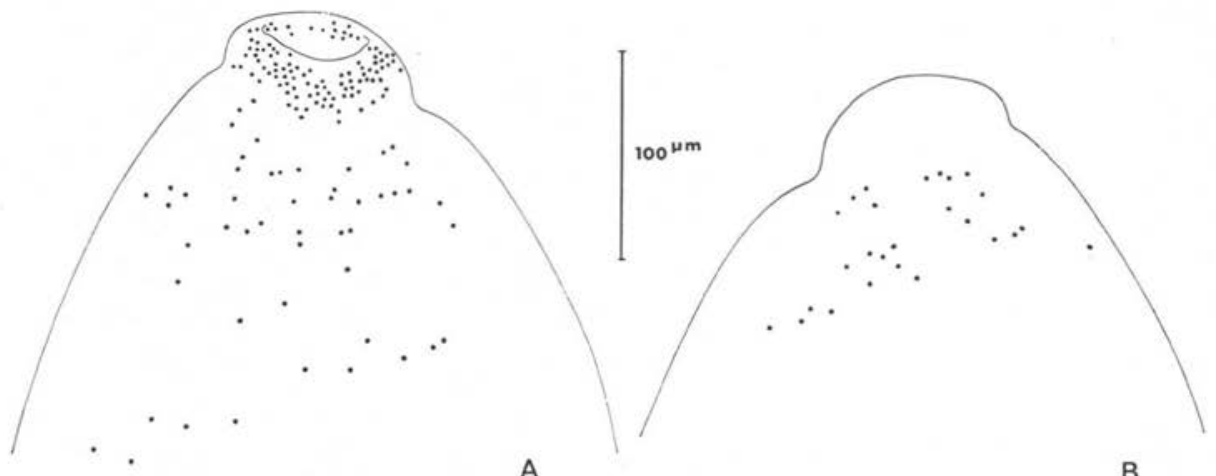

Fig. 2. Rédie-fille de F. hepatica, région antérieure. Chétotaxie. A : face présumée ventrale; B : face présumée dorsale.

\section{La cercaire.}

La souche de $F$. hepatica que nous étudions émet les cercaires à la lumière contrairement à la souche de $F$. gigantica utilisée par Albaret et coll. (1980) qui émet les cercaires à l'obscurité. Seules, les cercaires émises depuis peu de temps et nageant, montrent des sensilles après imprégnation; celles qui ont commencé à se fixer sur un substrat ou bien les métacercaires enkystées ne s'imprègnent pas.

a. Chétotaxie de la région céphalique (fig. 3 et fig. $6, D$ ).

Elle porte cinq cycles de papilles distribuées autour de la bouche ventrale. $\mathrm{C}_{1}$ porte 6 papilles, $\mathrm{C}_{\mathrm{II}}: 31$ à 36 papilles; $\mathrm{C}_{\mathrm{III}}: 22$ à 31 papilles; $\mathrm{C}_{\mathrm{IV}}: 12$ à 15 papilles ; $\mathrm{C}_{\mathrm{V}}$ : 9 à 13 papilles; au total la région céphalique porte 80 à 100 papilles, soit de chaque côté du plan de symétrie médio-saggital :

$$
\begin{aligned}
& \mathrm{C}_{1}=1 \mathrm{C}_{\mathrm{I}} \mathrm{V} \text { invaginée, } 2 \mathrm{C}_{\mathrm{I}} \mathrm{D} . \\
& \mathrm{C}_{\mathrm{II}}=2 \mathrm{C}_{\mathrm{II}} 1,2 \text { à } 3 \mathrm{C}_{\mathrm{II}} 2,2 \text { à } 3 \mathrm{C}_{\mathrm{II}} 3,9 \text { à } 10 \mathrm{C}_{\mathrm{II}} 4 . \\
& \mathrm{C}_{\mathrm{III}}=2 \mathrm{C}_{\mathrm{III}} 1,1 \text { à } 2 \mathrm{C}_{\mathrm{II}} 2,3 \mathrm{C}_{\mathrm{HI}} 3,9 \mathrm{C}_{\mathrm{III}} 4 . \\
& \mathrm{C}_{\mathrm{IV}}=2 \text { à } 3 \mathrm{C}_{\mathrm{IV}} 1,4 \text { à } 5 \mathrm{C}_{\mathrm{IV}} 2 . \\
& \mathrm{C}_{\mathrm{V}}=0 \text { ou } 1 \mathrm{C}_{\mathrm{V}} 1,2 \mathrm{C}_{\mathrm{V}} 2,3 \text { à } 5 \mathrm{C}_{\mathrm{V}} 3 .
\end{aligned}
$$

b. Région corporelle (fig. 4, A-G).

Aucune des cercaires observées ne montre une disposition vraiment symétrique des terminaisons sensorielles. 

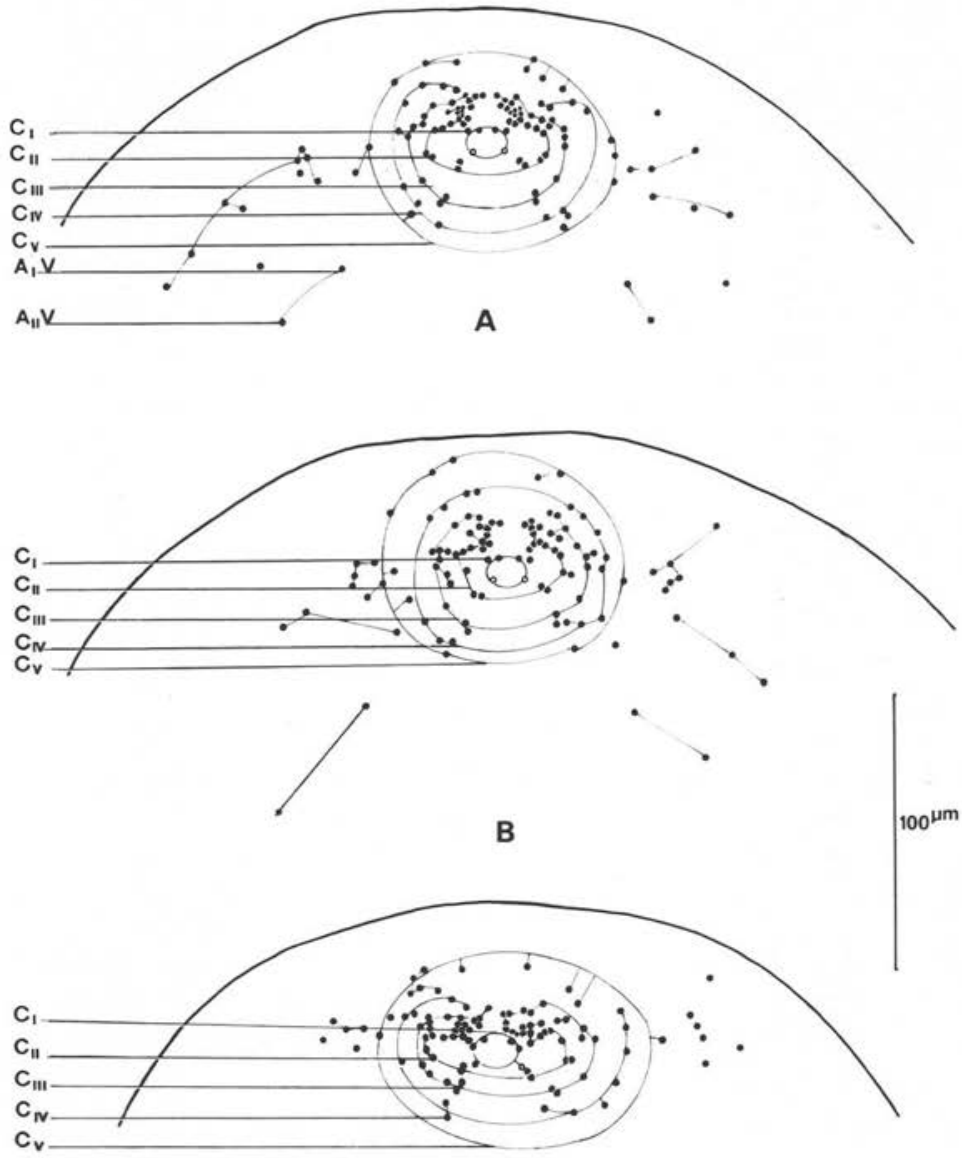

C

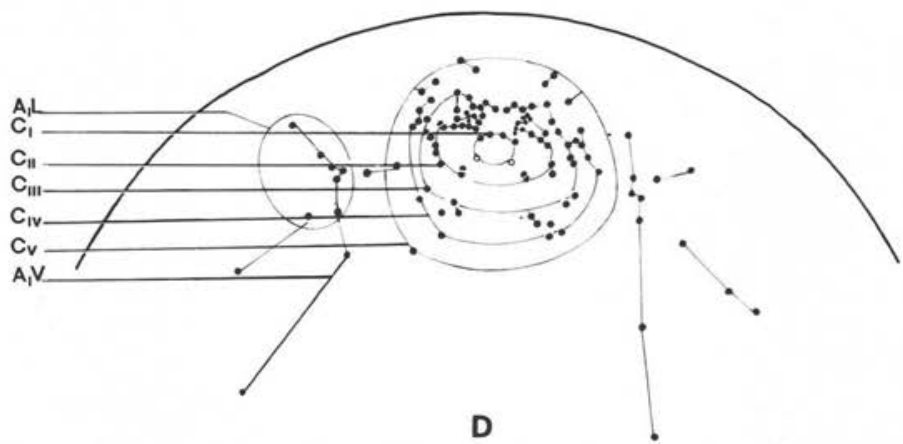

Fig. 3. Cercaire de $F$. hepatica. Chétotaxie céphalique. La papille $\mathrm{C}_{1} \mathrm{~V}$ invaginée est représentée par un cercle clair. 
- papilles latérales.

Elles sont comprises entre les niveaux $A_{I}$ et $A_{I I I}$; nous en comptons 8 à 15 par hémicorps.

- papilles ventrales.

Nous en comptons généralement 2 à 5 par hémicorps.

$\mathrm{A}_{\mathrm{I}}=0$ ou 1 à $2 \mathrm{~A}_{\mathrm{I}} \mathrm{V}$.

$\mathrm{A}_{\mathrm{II}}=0$ ou $1 \mathrm{~A}_{\mathrm{II}} \mathrm{V}$.

$\mathrm{A}_{\text {III }}=0$ ou $1 \mathrm{~A}_{\text {III }} \mathrm{V}$.

$\mathrm{P}_{\mathrm{III}}=1 \mathrm{P}_{\mathrm{III}} \mathrm{V}$.

- papilles dorsales.

$\mathrm{A}_{\mathrm{I}}=2$ à $3 \mathrm{~A}_{\mathrm{I}} \mathrm{D}$.

c. Région acétabulaire (fig. 4, H).

Sa chétotaxie est instable ; sur 49 cercaires, 26 n'ont pas de papille acétabulaire, 17 ont une seule papille, 3 ont deux papilles, 1 a trois papilles, 1 a une papille acétabulaire et une papille juxtacétabulaire, 1 a deux papilles juxtacétabulaires, soit :

$\mathrm{S}_{\mathrm{I}}=0$ ou 1 papille, exceptionnellement 2 ou 3.

d. Région caudale (fig. 5, fig. 6, F).

La queue porte six axes de papilles:

- deux axes ventraux UV de 16 à 22 papilles chacun ;

- deux axes latéraux UL de 10 à 17 papilles chacun : papilles $x$ de J. Richard (1971), auxquelles s'ajoute, près de l'extrémité caudale, une papille isolée : papille 1 de J. Richard ;

- deux axes dorsaux UD portant habituellement 3 papilles chacun, et plus rarement 2 ou 4.

\section{III. - Comparaison de F. bepatica et F. gigantica;}

\section{Discussion}

1. Le Miracidium (fig. $6, A, B, C$ ).

Chez $F$. hepatica, le nombre et la disposition des organites argyrophiles superficiels de la ceinture antérieure correspondent à ce qui a été décrit par Coe (1896), Wilson (1970), Køie et coll. (1976), mais il n'en est pas de même au niveau du térébratorium pour lequel les descriptions des auteurs divergent et diffèrent de nos observations. Nous pensons que de telles différences sont liées aux techniques utilisées. Quoi qu'il en soit, la comparaison des organites argyrophiles superficiels ne permet pas de distinguer le miracidium de Fasciola hepatica de celui de $F$. gigantica, qui a fait l'objet d'un travail récent (Albaret et coll., 1980). 


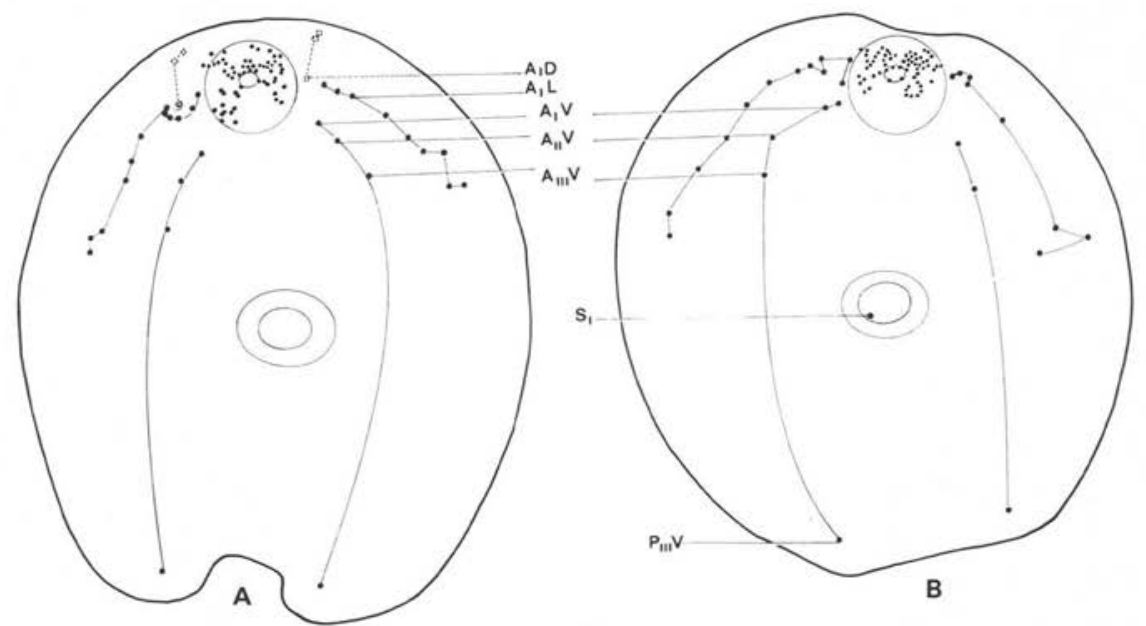

$100^{\mathrm{mm}}$
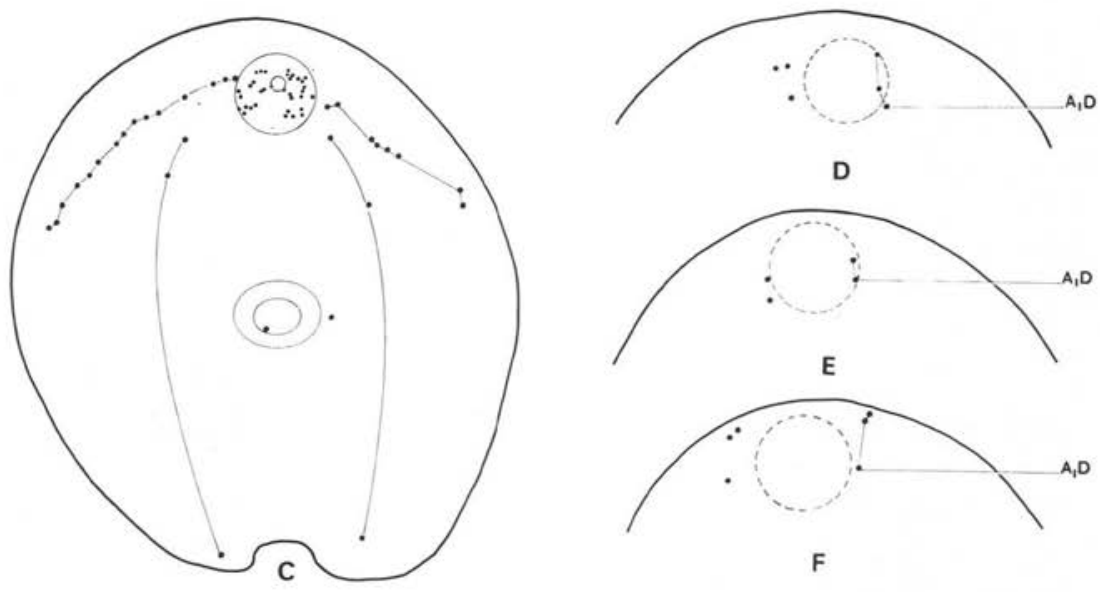

D
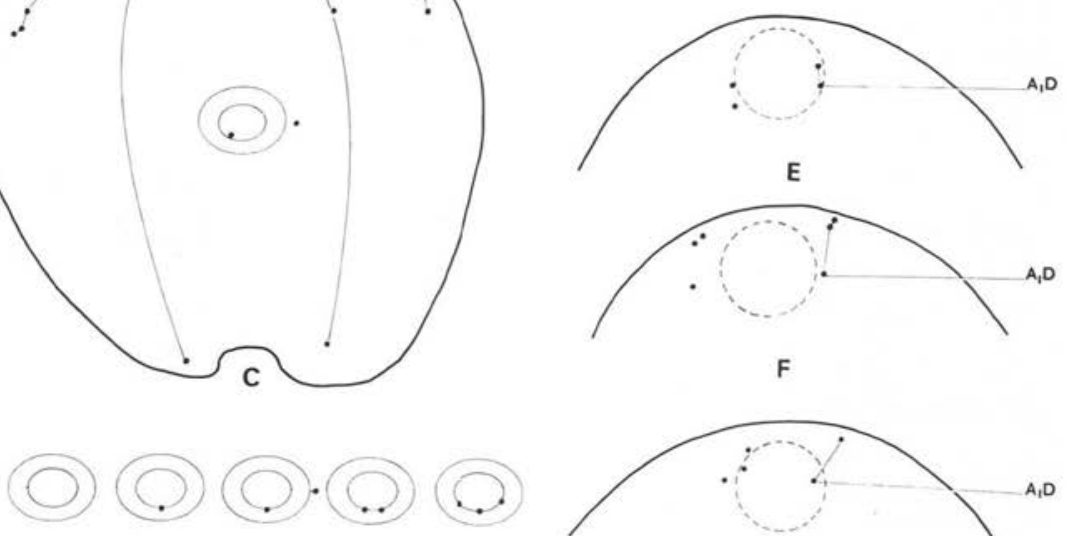

$\mathrm{H}$

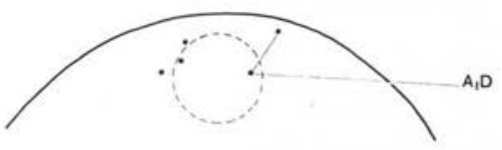

G

Fig. 4. Cercaire de F. hepatica. Chétotaxie. A, B, C : corps en vue ventrale; D, E, F, G : région antérieure du corps en vue dorsale; $\mathrm{H}$ : acétabulum. 
Le nombre et la disposition générale des plaques épidermiques ciliées du miracidium de $F$. hepatica sont conformes aux descriptions des auteurs (Coe, 1896, Mattes, 1949, Dawes, 1960, Southgate, 1970, Køie et coll., 1976). Cependant, selon Coe, la plaque médio-ventrale du deuxième étage est plus longue que les autres (fig. 1, planche 42); pour Køie et coll., elle s'insère entre les cellules du troisième étage. Comme nous l'avons vu, cette disposition n'est pas la règle générale et correspond à la position intermédiaire que nous avons observée chez un nombre réduit de miracidiums. Rappelons à ce propos que chez 62 à $82 \%$ des miracidiums, la plaque médioventrale du deuxième étage se prolonge jusqu'à la ceinture 3 . Or, chez le miracidium de $F$. gigantica, une telle disposition ne s'observe que dans 0 à $6 \%$ des cas, alors que dans 87 à $95 \%$ des cas la plaque médio-ventrale du deuxième étage s'interrompt au niveau de la ceinture 2 (Albaret et coll., 1980).

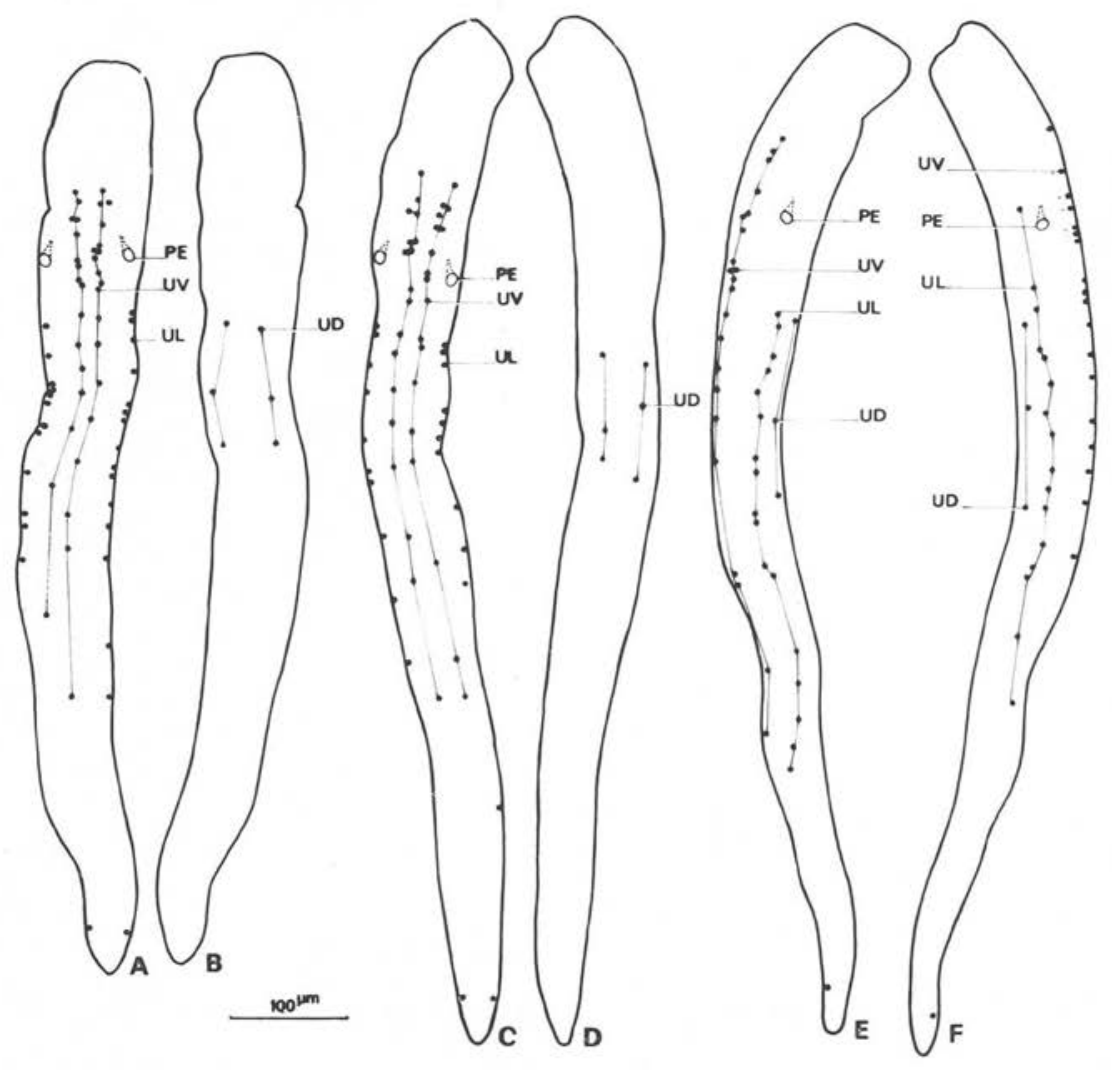

Fig. 5. Cercaire de F. hepatica. Chétotaxie caudale. A et $\mathrm{C}$ : papilles ventrales et latérales; $\mathrm{B}$ et $\mathrm{D}$ : papilles dorsales; $\mathrm{E}$ et $\mathrm{F}$ : papilles ventrales, latérales et dorsales. 


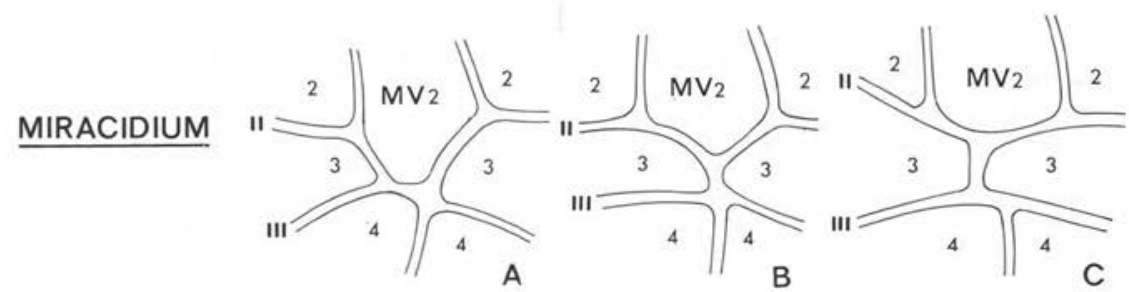

\begin{tabular}{|c|c|c|c|c|}
\hline \multirow{2}{*}{ F. hepatica } & France & $82 \%$ & $9 \%$ & $9 \%$ \\
\hline & Bulgarie & $62 \%$ & $20 \%$ & $18 \%$ \\
\hline & Souche 1 & $6 \%$ & $0 \%$ & $94 \%$ \\
\hline F. gigantica & Souche 2 & $4 \%$ & $9 \%$ & $87 \%$ \\
\hline Sénégal & Souche 3 & $0 \%$ & $5 \%$ & $95 \%$ \\
\hline
\end{tabular}

\section{CERCAIRE}

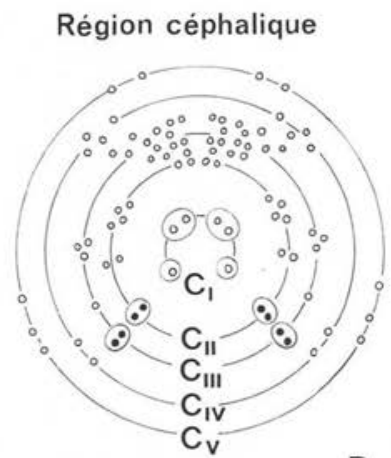

F. hepatica

D

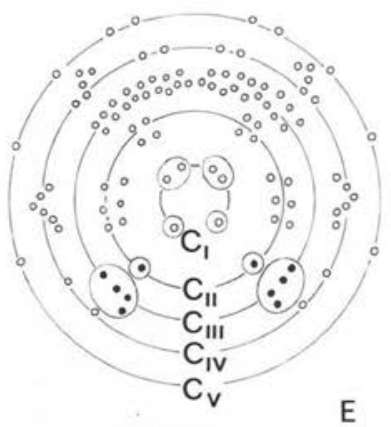

F. gigantica

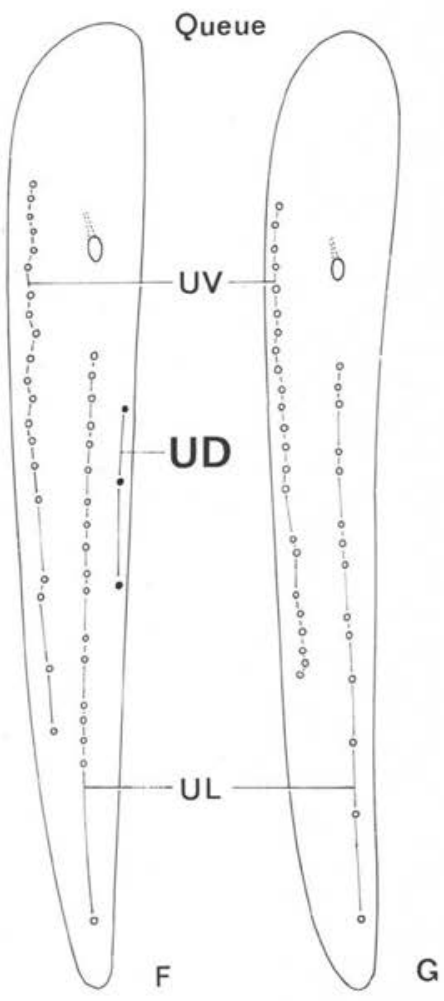

F. hepatica

F. gigantica

Fig. 6. Caractères comparés des miracidiums et des cercaires de Fasciola hepatica et $F$. gigantica. Miracidium: A, B, C. Variations de la disposition de la plaque ciliée médio-ventrale du deuxième étage (MV2); $2,3,4$ : plaques ciliées des deuxième, troisième et quatrième étages; II et III : deuxième et troisième ceintures. Cercaires : chétotaxies céphalique (D et E) et caudale ( $F$ et $G$ ). Les sensilles prises en considération pour la diagnose sont en noir. 
La disposition de la plaque ciliée médio-ventrale du deuxième étage (MV2) permet donc de distinguer statistiquement les miracidiums de chacune des deux espèces.

\section{La cercaire (fig. $6, D, E, F, G$ ).}

Les chétotaxies des cercaires de Fasciola hepatica et $F$. gigantica n'offrent à différents niveaux, ni régularité, ni symétrie parfaites; la composition de plusieurs groupes de papilles montre des variations relativement importantes; toutefois, dans la région céphalique, les niveaux $C_{1}, C_{11} 1$ et $C_{111} 1$ paraissent stables. Le cycle $C_{1}$ est identique pour les deux espèces et ne permet pas de les différencier; les papilles composant $\mathrm{C}_{\mathrm{II}} 1$ et $\mathrm{C}_{\mathrm{II}} 1$ sont en nombre différent pour chaque espèce ; ce nombre est assez constant et permet de reconnaître une cercaire de $F$. hepatica d'une cercaire de $F$. gigantica. Chez $F$. hepatica, $\mathrm{C}_{\mathrm{II}} 1$ porte deux papilles accolées ou très proches ; chez $F$. gigantica, $\mathrm{C}_{\mathrm{II}} 1$ porte une seule papille; chez $F$. hepatica, $\mathrm{C}_{\mathrm{II}} 1$ porte deux papilles accolées ou très proches; chez $F$. gigantica, $\mathrm{C}_{\mathrm{II}} 1$ porte un groupe de quatre (parfois cinq) papilles plus ou moins rapprochées (fig. $6, D, E$ ). La région caudale permet aussi de reconnaître une espèce de l'autre : $F$. hepatica montre six axes de papilles caudales et en particulier deux axes dorsaux UD ; F. gigantica montre quatre axes et n'a pas d'axes dorsaux (fig. 6, F, G). D'après les conclusions de J. Richard (1971) et de l'un d'entre nous (Ch. Bayssade-Dufour, 1979), ce caractère laisse à penser que la forme à six axes est plus archaïque que celle à quatre, donc que $F$. gigantica dériverait de F. hepatica.

REMERCIEMENTS. - Nous remercions le Docteur Poulain, Directeur du Service sanitaire de l'Abattoir de Versailles, qui nous a procuré les douves adultes, et le Docteur Viyatchev qui nous a apporté des douves de l'Abattoir de Sofia.

\section{Bibliographie}

Albaret J.-L., Bayssade-Dufour Ch., Diaw O.-T., Vassiliadès G., Gruner L.: Données complémentaires sur les organites argyrophiles superficiels du miracidium et de la cercaire de Fasciola gigantica, Cobbold, 1855 (Trematoda: Fasciolidae) et sur l'épidémiologie de ce parasite. Ann. Parasitol. Hum. Comp., 1980 (sous presse).

Azimov Sh., Nazarov A. N. : Study on the species composition of Fasciola (F. gigantica, $F$. hepatica and mixed forms) in sheep and cattle in the Uzbek SSR. Gel'minty pischevykh produktov. Tezisy dokladov mezhrespublikanskoi nauchnoi Konferentsii, 1972, 22-25 (en russe).

Bayssade-Dufour Ch. : L'appareil sensoriel des cercaires et la systématique des Trématodes digénétiques. Mem. Mus. Nat. Hist. Nat., Paris, sér. A. Zool., 1979, 113, 1-81.

Bhalerao G. D.: The common worms of sheep and goats in India and their control. Agric. Livestock India, 1934, 4, 655-669.

Boray J. C.: Studies on the relative susceptibility of some lymnaeids to infection with Fasciola hepatica and Fasciola gigantica and on the adaptation of Fasciola spp. Ann. Trop. Med. Parasitol., 1966, 60, 114-124.

Coe W. R. : Notizen über den Bau des Embryos von Distomum hepaticum. Zool. Jb. Abt. Ant. Ontog., 1896, 9, 561-570. 
Combes C., Bayssade-Dufour Ch., Cassone J.: Sur l'imprégnation et le montage des cercaires pour l'étude chétotaxique. Ann. Parasitol., Hum. Comp., 1976, 51, 399-400.

Dawes B.: A study of the miracidium of Fasciola hepatica and an account of the mode of penetration of the sporocyst into Limnaea truncatula. Libro homenaje al Dr. Eduardo Caballero y Caballero. Escuela Nacional de Ciencias Biologicas, 1960, 95-111.

Everaert G.P. J., Jawhari M., Gaufreteau A.: De la présence de grandes douves Fasciola gigantica sur des foies d'asins au Maroc. Rev. Med. Vet. Toulouse, 1974, 125, n.s., 541-544.

Kendall S. B.: Relationships between the species of Fasciola and their Molluscan Hosts. Adv. Parasitol., 1965, 3, 59-98.

K $\phi$ ie M., Christensen N. O., Nansen P.: Stereoscan studies of eggs, free-swimming and penetrating Miracidia and early Sporocysts of Fasciola hepatica. Z. Parasitenk, 1976, 51, 79-90.

Lin Y.K., Sung Y.L., Chen T. J., Chen D.H.: Studies on the development of Fasciola gigantica Cobbold in its snail host and the epidemiology of fascioliasis of cattle in the Tsinchiang Region, Fulien. Acta Zool. Sinica, 1974, 20, 378-394 (en chinois, résumé anglais).

Mattes O.: Wirtsfindung, invasionsvorgang und wirtsspezifität beim Fasciola miracidium. Z. Parasitenk, 1949, 14, 320-363.

Nasir P., Diaz M. T. and Lemus de Guevara D. : Studies on freshwater larval Trematodes. XIX. Two species of Gymnocephalic Cercariae from Venezuela. Zool. Anz., 1968, 181, 427-434.

Popov A.: La distomatose en Bulgarie. Bull. Off. Intern. Epizooties, 1957, 48, 496-502.

Porter A.: The larval trematoda found in certain South African mollusca with special reference to schistosomiasis (bilharziasis). South Afric. Inst. Med. Res., 1938, 1-492.

Richard J.: La chétotaxie des cercaires. Valeur systématique et phylétique. Mém. Mus. Nat. Hist. Nat., 1971, série A, Zool. 67 : 1-179.

Sahba G.H., Arfaa F., Farahmandian I., Jalali H.: Animal fascioliasis in Khuzestan, Southwestern Iran. J. Parasitol., 1972, 58, 712-716.

Sazanov A.M.: The susceptibility of molluscs to infection with Fasciola hepatica and Fasciola gigantica. Materialy Nauchnoi Konferentsii Vsesoyuznogo Obshchestva Gel'mintologov (Trematody $i$ Trematodozy), 1977, 29, 128-133 (en russe).

Southgate V.R.: Observations on the epidermis of the miracidium and on the formation tegument of the sporocyst of Fasciola hepatica. Parasitology, 1970, 61, 177-190.

Wilson R. A. : Fine structure of the nervous system and specialized nerve endings in the miracidium of Fasciola hepatica. Parasitology, 1970, 60, 399-410. 